\title{
Discussion on Conceptual Design in Fantasy Film
}

\author{
Yu Guo \\ Film-Video-Animation School \\ Sichuan Fine Arts Institute \\ Chongqing, China
}

\begin{abstract}
Film is called "dream machine". Fantasy films show magical worlds for us. With development of science and technology, these films attract people's attention through depending on bold imagination and magnificent picture effect. The "conceptual design" at earlier stage of films plays an important role in these works. It has very important reference significance on film production through understanding related knowledge on "conceptual design".
\end{abstract}

Keywords-movie animation; world view; 5w1h; conceptual design

\section{INTRODUCTION}

Each film animation work tries to bring different audio visual experience for audiences. Fantasy film works pay more attention to "novel", "magical" and "unique", namely whether the theme is novel, whether the effect is magical and whether the modeling is unique. In order to realize these purposes, at earlier stage of movie animation production, it is necessary to carry out complete planning and design. Conceptual design is the most important part of it.

Fantasy film production in foreign countries attaches great importance to conceptual design. At earlier stage of production of each fantasy film, quite a few concept designers put forward numerous conceptual design scheme and plan concrete contents such as story line, role and scene through it. The film of The Matrix is a successful example. It shows fantastic imagination of people for space-time concept in the future and a virtual world formed by numbers and procedures. Because of describing existence and nothingness in detail, this film even causes thinking on philosophy. Before several years when the film starts to shoot, directors the Wachowski Brothers begin to design the whole story and draw a good deal of sketch of conceptual design. After long-playing preparation, The Matrix renounces the world splendidly and becomes landmark wok in development history of film industry. "Fig. 1"

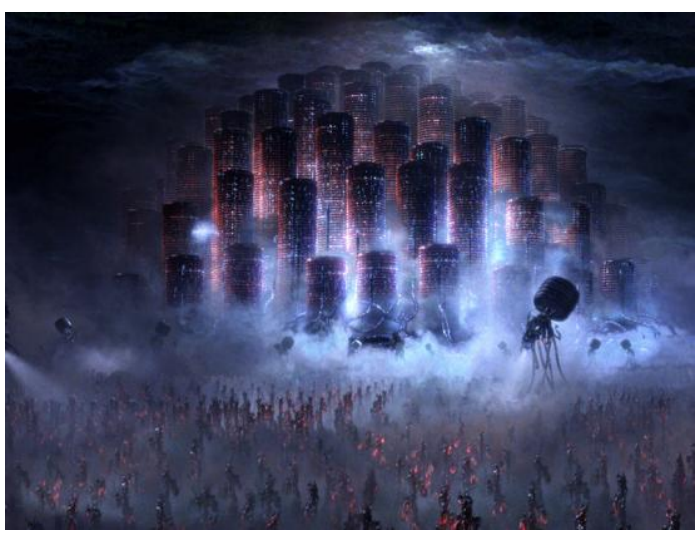

Fig. 1. Picture in The Matrix

Conceptual design has long history in western countries. From The Republic of Plato to Utopia of Moore, we can find conceptual design in them. With development of times, conceptual design is applied to film production field as well as development and production of computer games.

\section{CONCEPTUAL DESIGN Is ENFORCEABLE SYSTEMATIC PLANNING}

Concretely speaking, conceptual design in films means planning general background of the film and operational relation between elements under this background. We can summarize it into " $5 \mathrm{~W} 1 \mathrm{H}$ " of conceptual design.

The first "W" is "World View". Like computer games, "World View" stipulates types of animation - real world based on reality, or surreal science fiction world or unreal virtual world. Real world follows existing scientific law and social operation law; science fiction world shall follow existing scientific law or science and technology through strict reasoning; virtual or magic world requires people to create and the creation must have reasonable explanation.

In fantasy films, science fiction and fantasy are different types. Science fiction films base on available knowledge or reasonable speculation on the basis of available knowledge. It even needs the support of relatively forward-looking theories. The contents mainly show future world.

Theoretical basis of science fiction film is science futurology. The new-rising subject at the beginning of last century is a scientific, systematic and comprehensive knowledge that explores common fate of human. It sounds the 
alarm, or depicts blueprint for people and makes people face the future selectively through scientific analysis and in-depth exploration. Therefore, a lot of science fiction films have certain warning significance. From Water world, We see predicament of people after losing homeland on the earth. From Deep Impact, we see threat of minor planet on earth. From The Day after Tomorrow, we also feel disasters brought by climate change for people.

Because the surreal world view shall be scientific, it will change with scientific development and development of times. For example, in the era of Jules Verne, the travel from the earth to the moon can only be a fantasy. Jules Verne designs a huge cannon standing in Florida and launches a cannonball that carries three people to the moon at the initial speed of 12,000 feet per second. In order to ensure that the plot is rigorous, Jules Verne invites his elder male cousin, professor of higher mathematics, to calculate data of ballistic curve in person, in order to let this classic base on science. But nowadays, the travel to moon with rocket motor as the impetus is no longer difficult thing. Science fiction creation needs to choose new engine and transportation machine, nuclear engine, interstellar ramjet, antimatter engine, ion engine and photon engine. People even think of using light pressure of fixed star and stellar wind to sail in the universe. However, when all of these realize, science fiction creation needs to speculate according to scientific and technological level at that time...

This kind of surreal world view is often called "fantasy". The most prominent feature of this kind of works is "fictional universe". Such as series of The Lord of the Rings, the "middle earth" that the story takes place is not real. Everything in it is also designed by Tolkien many years ago and improved continuously and can continue to move forward. In this kind of world view, directors play the role of God, to create geographical environment, climate, race and culture, etc. All base on conception of people. "Fig. 2"

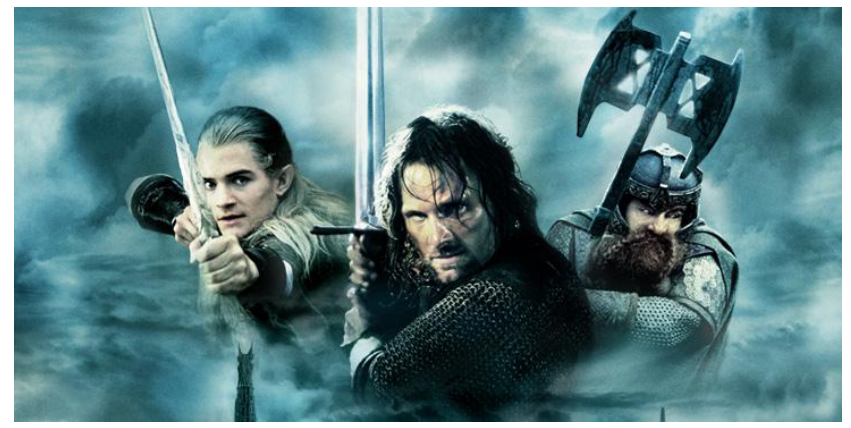

Fig. 2. Picture in The Lord of the Rings

In western countries, another famous surreal world view system is "Eberron", which is a huge world including "Dungeons and Dragons". People adapt this work and publish more than four hundred readings. In 1980s, it was made into TV play. Afterwards, it was adapted into famous roles play games in Europe and America. The sales volume of related products around the world exceeds one billion U.S. dollars. In this world, monsters infest. Magic occupies important position. The film of Dungeons and Dragons adapted has spectacular scene. Corpse king and monsters enter on the stage. The scene that the dragon destroys the city in the end is striking. "Fig. 3"

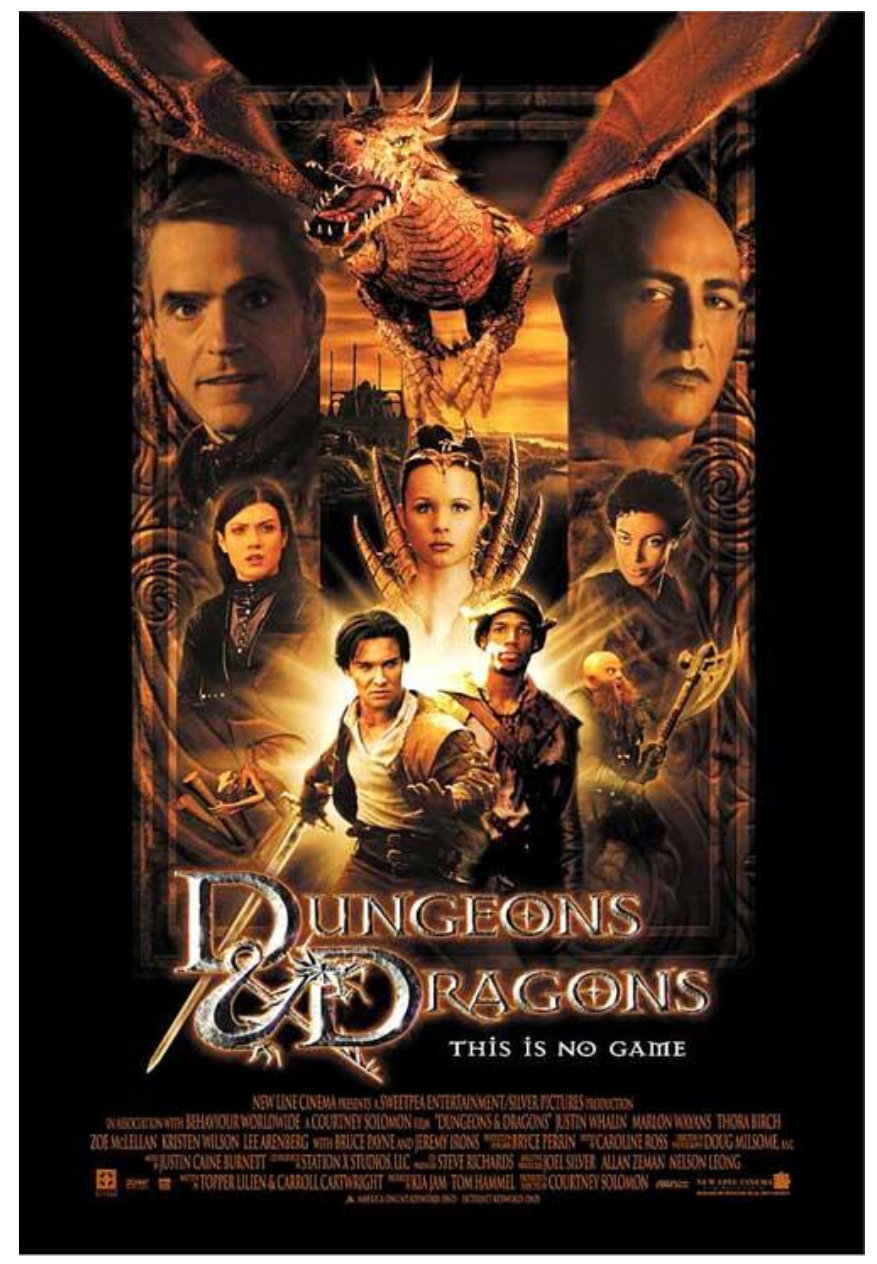

Fig. 3. Picture in film of Dungeons and Dragons

Imagination in eastern culture is richer. Shan Hai Jing, Journey to the West and The Legend of Deification are great fantasy themes. Many movie animation works adapted according to them also receive more and more attention from people.

The second "W" is "When". It gives time background inside the film. "When" includes big time scale, such as century, generation and period, as well as small time scale, such as morning, midnight and afternoon. Conceptual design shall consider concrete characteristics of each time. When necessary, show it through appropriate means.

The third "W" is "Where". It gives space background of fantasy film. Does it occur in seaside or high mountains, desert or polar region, outer space or earth core? Each scene has characteristics of its own. In conceptual design, we shall pay close attention to differences between different regions and different spaces and relations between different elements in different scenes and show them.

The fourth "W" is "Who". It discusses the protagonist of movie animation, people or machine, elf or monster? Each has habits and hobbies of its own. Conceptual design shall take 
characteristics of roles into full consideration and even write resumes of roles.

The fifth "W" is "Why". Why shall we design in this way? Is there any better image or better way? Is there any better story? No matter in preparation at earlier stage or in the process of conceptual design, or in post production, we shall always ask ourselves "why". If finding better conception in the process of medium-term production, we need to change if condition permits.

The "H" is "How". For example, you design an alien monster for an animation. Through the previous thinking, we have imagined geographic position of it - bog close to polar region on z planet in k galaxy. Next we shall consider: what it will eat? What kind of ways will it use to hunt? Does it have natural enemy? How does it sleep? How does it communicate with the same kind? We shall pay attention to these contents.

We can see that in fantasy film, the most important part is setting of "world view". It defines basic style of movie animation. It is also the contents that fantasy film writers want to convey to audiences and let audiences approve. World view of fantasy films takes real world as reference. Some base on myths spread in ancient times. For example, The Lord of the Rings bases on fairy story that passes on from generation to generation in Northern Europe.

\section{COMPLICATION OF CONCEPTUAL DESIGN}

In fantasy films, conceptual design involves many aspects, such as role modeling, clothing modeling and props design, scene design. Conflicts shall not exist between different elements. For example, you conceive there is dinosaur with two feet that has been domesticated for people to ride, so it shall have rapid movement, endurance, easy to control. A series of related designs are involved: harness design on dinosaur (not influence advancement of dinosaur, but easy for controller to rein); clothing design of controlling. But if you change your mind and choose four-legged dinosaur that eats grass for people to ride, how can you change related parts?

Complication of conceptual design also shows that each concrete design object shall have details as many as possible. Conceptual design in films cannot only be satisfied with introducing a vague idea. It shall have concrete forms and convincing details of design as many as possible. Designers who know mechanical design shall know that the setting of air vehicle is not merely an appearance design. He must consider its dynamical system, control system, command system, lifesaving system and combat system. The form and use of each concrete part shall be able to stand the test. In Howl's Moving Castle of Miyazaki Hayao, the protagonist Howl lives in a moving castle, which has complicated internal space and complete facilities. In Studio Ghibli, this illusory castle has complete design. Even rooms that cannot be seen in the film have plane graph, vertical view and detailed drawing of local details.

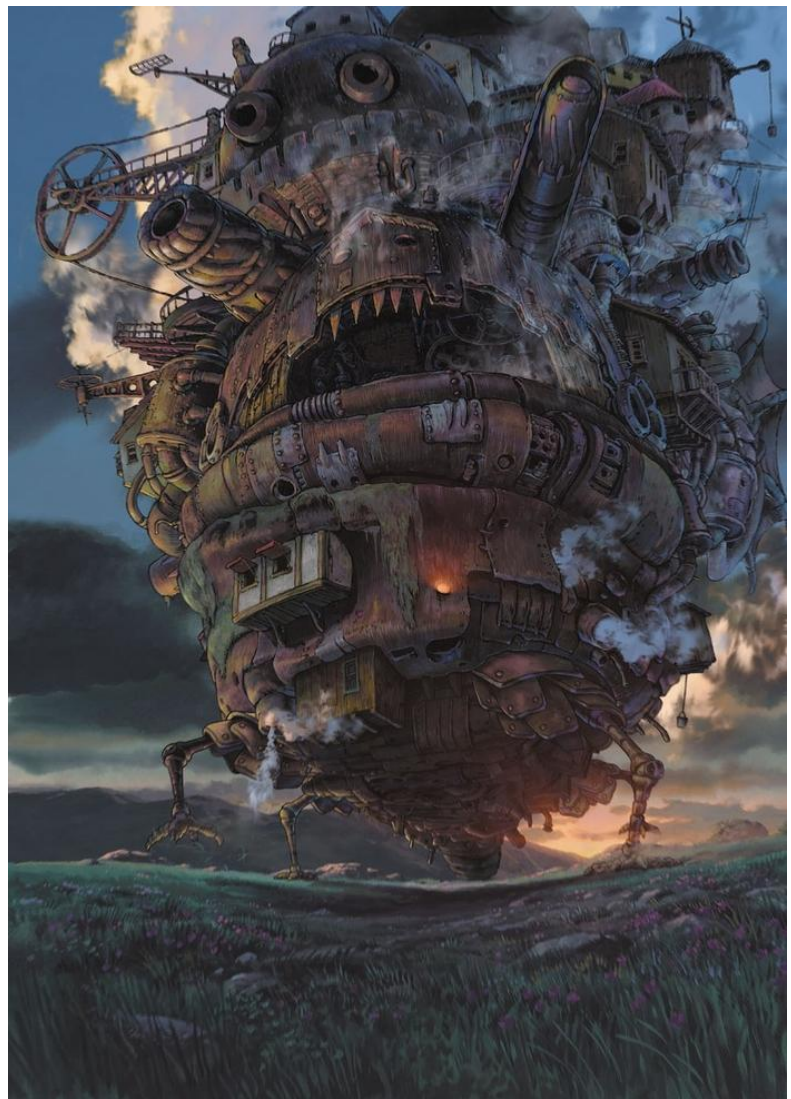

Fig. 4. Picture in Howl's Moving Castle

The bigger the world, the more complicated the conceptual design required will be. The series of The Lord of the Rings include nine main races. Each race has different life, customs and myths, clothing even martial arts. Tolkien began to create novels of Hobbit and trilogy of The Lord of the Rings between 1930 and 1947. After introduction in 1960s, they attract numerous readers. Because of limitation of shooting technique, until 1993, Peter Jackson, Hollywood director and fan of The Lord of the Rings decided to invest 300 million dollars to shoot series of The Lord of the Rings. At earlier stage of shooting, conceptual designers including Alan Lee and John Haowei make numerous drawings for the film. It makes series movie of The Lord of the Rings full of striking visual effect. "Fig. 5"

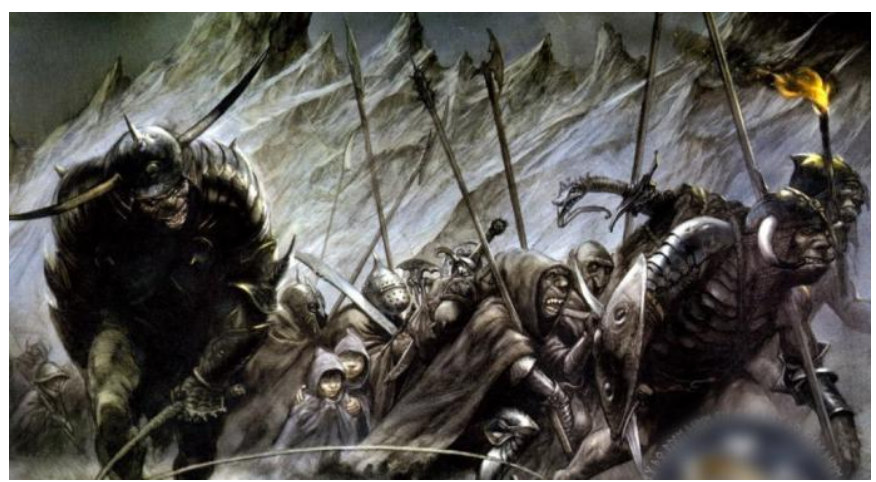

Fig. 5. The Lord of the Rings 


\section{Conceptual Design Is UniQue}

Unusual visual perception comes from unique design. Fantasy films always want to show the most striking scene to audiences and leave them the most profound impression. It requires conceptual designers to shape particular effects from many aspects.

\section{A. Fresh Pattern}

Innovation on pattern may be the most eye-catching. Many conceptual designers are willing to put forward unprecedented conception, such as automobile design in series movie of 007 . In "Fig.6", the appearance does not have big breakthrough. People are interested in its excellent driving performance and super strong multifunctional design. In Sky Captain and the World of Tomorrow, the old fighter piloted by the captain rushes from the sky in the sea. Under water, the covert screw propeller stretches out and becomes a submarine... Innovation on pattern brings fresh experience for us. "Fig.7"

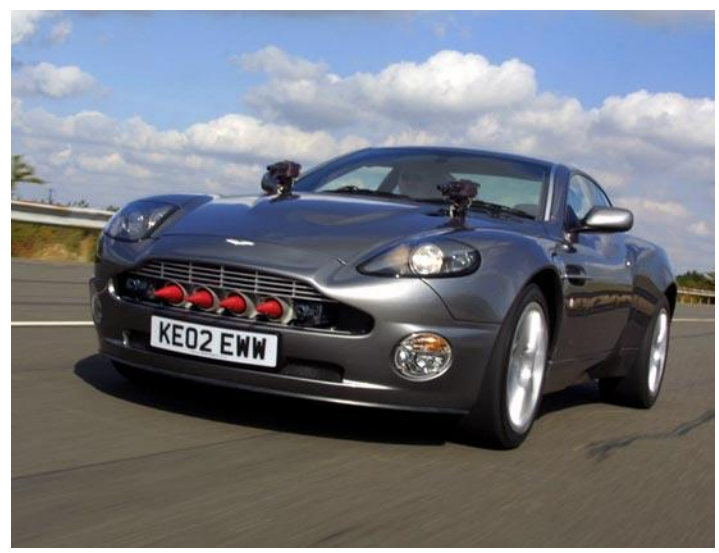

Fig. 6. Automobile design in film series of 007

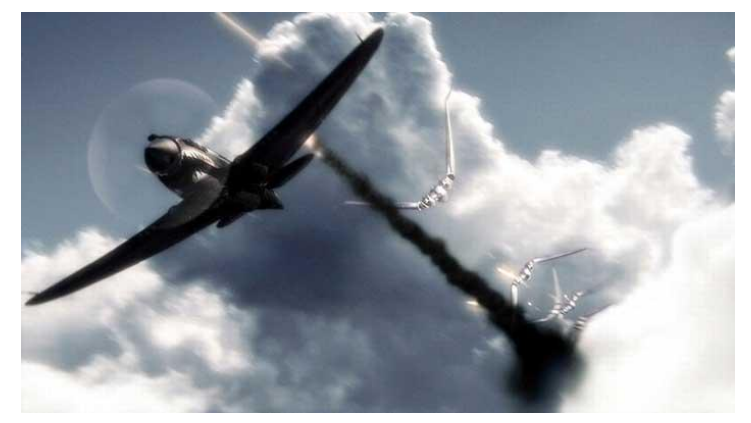

Fig. 7. Old fighter in film of Sky Captain and the World of Tomorrow

\section{B. Unique Modeling}

Films are audio-visual arts. Some parts of visual impart come from unique modeling in films. From alien creature in Alien to machine octopus in The Matrix, from universe fleet in Star Wars to underground palace in Myth, each role and each scene contains great efforts devoted by conceptual designers.

However, some conceptual designers excessively pursue visual effects but neglect inner rationality, which is the problem that designers should pay attention to in conceptual design.
Conceptual design does not get enough attention in domestic film industry. It is because great gaps exist between foreign countries and our country in creation of fantasy films. Designers lack enough understanding of conceptual design. Most designers of movie animation pay attention to imitation of painting style of foreign masters. With maturity of movie animation industry, it is believed that more and more people will engage in conceptual design of films and bring surprise for us in the field of fantasy film.

\section{REFERENCES}

[1] Han Xiao. Movie Animation Scene Design [M], Beijing: China Ocean Press, 2005.

[2] Lu Tao. Film Language [M], Xi'an: Shanxi People's Fine Arts Publishing House, 2003.

[3] Zhang Renfeng, Yu Jianhong. English-Chinese Chinese-English Movie Dictionary [M], Beijing: China Film Press, 2000. 